\title{
Curved apparent motion induced by amodal completion
}

\author{
Sung-Ho Kim • Jacob Feldman • Manish Singh
}

Published online: 9 November 2011

(C) Psychonomic Society, Inc. 2011

\begin{abstract}
We investigated whether amodal completion can bias apparent motion (AM) to deviate from its default straight path toward a longer curved path, which would violate the well-established principle that AM follows the shortest possible path. Observers viewed motion sequences of two alternating rectangular tokens positioned at the ends of a semicircular occluder, with varying interstimulus intervals (ISIs; 100-500 ms). At short ISIs, observers tended to report simple straight-path motion-that is, outside the occluder. But at long ISIs, they became increasingly likely to report a curved-path motion behind the occluder. This tendency toward reporting curved-path motion was influenced by the shape of tokens, display orientation, the gap between tokens and the occluder, and binocular depth cues. Our results suggest that the visual system tends to minimize unexplained absence of a moving object, as well as its path length, such that AM deviates from the shortest path when amodal integration of motion trajectory behind the curved occluder can account for the objective invisibility of the object during the ISI.
\end{abstract}

Keywords Apparent motion - Occlusion - Amodal completion · Tunnel effect

In a cluttered environment of opaque objects and surfaces, occlusion of an object behind another is ubiquitous (Gibson, 1979). Occlusion poses a difficult challenge to the visual system because retinal projection of physically

S.-H. Kim $(\bowtie) \cdot$ J. Feldman $\cdot$ M. Singh

Department of Psychology and Center for Cognitive Science,

Rutgers University,

New Brunswick, NJ, USA

e-mail: sungho4@eden.rutgers.edu continuous objects does not provide local sensory information corresponding to parts hidden behind occluding surfaces. However, we do not have the impression as we view the world that it is filled with object fragments; rather, the objects that we see appear to be complete ones, a phenomenon called amodal completion or amodal continuation (Kanizsa, 1979; Michotte, Thinès, \& Crabbé, 1964/ 1991; Shimojo \& Nakayama, 1990).

Amodal completion in static scenes have been extensively studied (e.g., Anderson, Singh, \& Fleming, 2002; Bruno, Bertamini, \& Domini, 1997; Gerbino \& Salmaso, 1987; He \& Nakayama, 1994; Kanizsa \& Gerbino, 1982; Kellman \& Shipley, 1991; Rauschenberger, Peterson, Mosca, \& Bruno, 2004; Sekuler, Palmer, \& Flynn, 1994; Singh, 2004; Watanabe, 1995). But the need for amodal completion is even more pervasive in a dynamic setting (Gibson, 1979). A moving object disappears and reappears as it passes behind other surfaces, or covers and uncovers others when it moves in front. Moreover, stationary objects are frequently occluded by an intervening surface due to the change in an observer's viewpoint, since mobile observers are able to move their eyes, heads, and bodies. ${ }^{1}$ However, as compared with the static case, dynamic amodal completion has been investigated relatively little.

One of the most famous demonstrations of amodal continuation in dynamic scenes is the tunnel effect (Burke, 1952; Michotte et al., 1964/1991; Wertheimer, 1912/1961). Suppose that a moving object gradually disappears next to a large stationary screen (tunnel), and then another similar

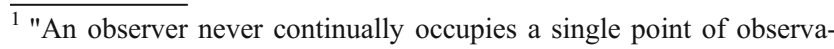
tion except under special circumstances such as death" (Clocksin, 1980).
} 
object appears on the other side of the screen. In this situation, object motion is temporarily invisible between the end of the first (pretunnel) and the beginning of the second (posttunnel) object motion. Nonetheless, observers unanimously report (under the appropriate spatiotemporal display conditions) that a single object moves behind the tunnel, suggesting that the representation of the moving object is amodally maintained during its disappearance, which links the two spatiotemporally discontinuous visible motion phases. Furthermore, Burke and Michotte et al. found that people could perceive not only the presence of an amodally moving object, but also its location and motion-related properties behind the tunnel, such as apparent speed and trajectory of the invisible motion. Strikingly, even when the posttunnel object path was not a linear continuation of the pretunnel object path, such that the two paths formed an angle less than $180^{\circ}$ but were co-circular at the entering and exiting points, observers could still perceive the continuity of movement behind the tunnel, via a relatively long, curved trajectory connecting two visible object motion phases (Burke, 1952; Michotte et al., 1964/1991).

Most classical and recent studies on the tunnel effect have used displays of continuously moving objects (e.g., Burke, 1952; Flombaum \& Scholl, 2006; Kawachi \& Gyoba, 2006). On the other hand, in an apparent motion (AM) display where two motion tokens are successively flashed next to a screen on opposite sides, the tunnel effect can be considered as an AM of a momentarily occluded object, in which the first token represents a pretunnel object and the second token represents a posttunnel object whose connection is perceived via perceptual filling in of the apparent path behind the screen (Wertheimer, 1912/1961). ${ }^{2}$ In addition, AM studies have shown that a momentarily disappearing object is perceived as persisting behind an occluding surface across time (e.g., Anstis \& Ramachandran, 1985; Blake, Ahlström, \& Alais, 1999; Ramachandran, Inada, \& Kiama, 1986; Sigman \& Rock, 1974; Yantis, 1995). If an AM display can elicit the percept of amodal continuation of motion trajectory in the presence of an occluding surface, can the occluded motion deviate from the straight path under certain conditions, as in the curved tunnel effect (Burke, 1952)?

In a standard AM display of two alternating dots, observers typically report seeing the shortest possible path between the two dot positions, which is generally a straight line, although there are infinitely many possible paths along which an object may move from one position to another. To

\footnotetext{
${ }^{2}$ In fact, the original finding of the tunnel effect by Wertheimer came from his studies on AM, but not continuous motion, and the term, tunnel effect, originated from a verbal report from one of his observers, who described an AM whose path was hidden by a screen "as if I saw the motion going through a tunnel" (Wertheimer 1912/ 1961, p. 1062).
}

elicit a longer, nonlinear curved AM that overcomes the default bias for the shortest path, other settings are required for inducing extra momentum to the direction of curved path. Such conditions include the presence of an obstructing object or surface intervening in the space along the shortest path (Kolers \& Pomerantz, 1971), an orientation difference between the motion tokens (Foster, 1975), priming of a curved gray path during the interstimulus interval (ISI) (path-guided motion; Shepard \& Zare, 1983), embedding motion tokens in a context of a dot field moving along V-shaped trajectory (entrained motion; Anstis \& Ramachandran, 1986), and biophysical constraints on motion (Shiffrar \& Freyd, 1990).

In the present study, we investigated whether the shortest possible path constraint can be overcome by amodal integration of the motion trajectory behind a curved occluder, which is compatible with a longer detour route. We presented our observers AM sequences containing two small rectangular shapes alternately abutting each end of a semicircular occluder (Fig. 1; an animated version of Fig. 1 can be viewed at http://rci.rutgers.edu/ sungho4/curvedAM. $\mathrm{htm}$ ). In addition, by design, the occluder resembled a "tube" through or behind which a moving object might pass (see the General Method section). We chose this stimulus arrangement, in part, because we hypothesized that the apparent physical plausibility of the motion through or behind it might facilitate the percept of curved-path motion.

The temporal interval between successively flashing tokens is an important factor for a longer, curved-path AM. In AM displays that possibly elicit curved motion, the shortest trajectory is preferred at short temporal intervals (i.e., stimulus onset asymmetry [SOA]; or ISI, if stimulus duration is fixed) between motion tokens, but longer curved motion tends to be experienced more at relatively long temporal intervals than at short ones (e.g., Kolers \& Pomerantz, 1971; Shepard \& Judd, 1976; Shepard \& Zare, 1983; Shiffrar \& Freyd, 1990). In addition, Burke (1952) reported that in the above-mentioned curved tunnel-effect display, the amodal trajectory behind an occluder varied as a function of the interval of the invisible phase, which is analogous to temporal separation between alternately flashing tokens in an AM display. When the interval was relatively long, the hidden trajectory appeared as a curve, of varying complexity, but when the interval was short, the hidden trajectory was the simple rectilinear continuation of the two exposed trajectories.

We were mainly interested in the effect of the length of temporal separation (ISI) on the perceived path of AM, because if our AM display generates the percept of curvedpath motion behind the semicircular occluder, the ISI between successively flashing tokens corresponds to the duration of occluded motion. In a standard two-flash AM display, at a relatively short temporal separation, alternately 
Fig. 1 a Illustration of the stimuli used in Experiment 1. A motion token is presented for a token duration (TD) at one end of a stationary semicircular tube, then disappears during an interstimulus interval (ISI) before it reappears at the opposite end of the tube, and so on. b Two possible paths of the AM, straight and curved ones. c Spatiotemporal diagram of the stimulus sequence shown in panel a. Time forms the third dimension in depth, along with the $x-y$ picture plane. A shaded bar abutting the first two motion tokens in space-time represents unexplained absence of a visible object during the ISI for straightpath motion interpretation a

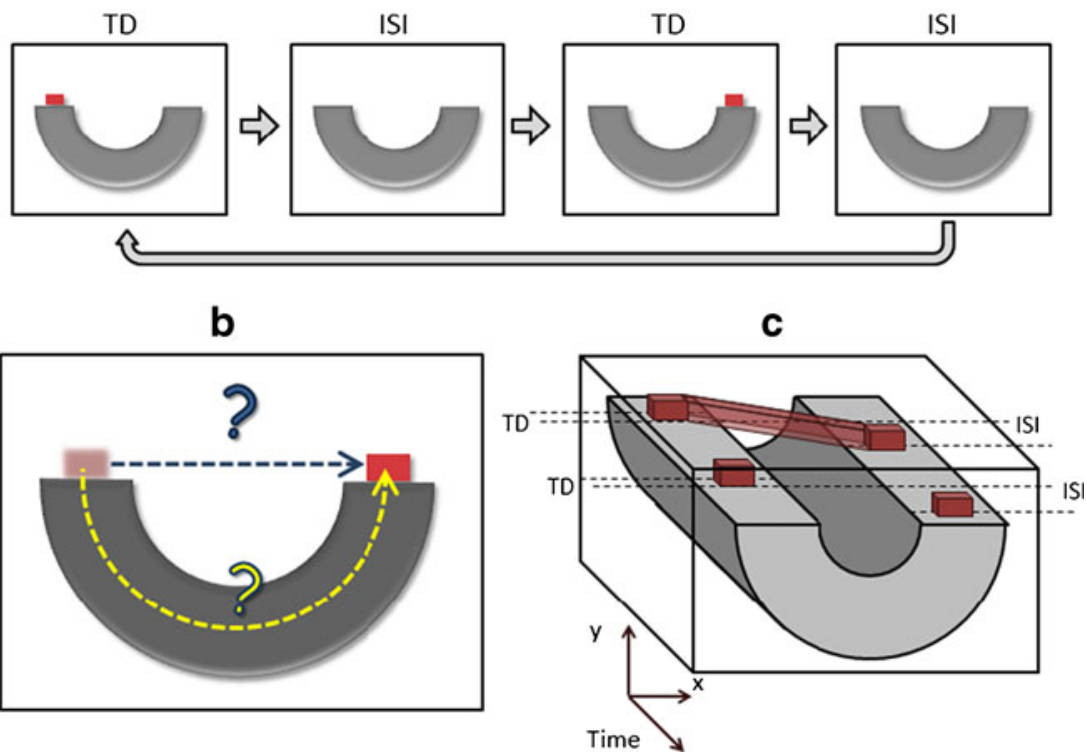

flashing objects will generate the percept of a persisting object moving over the most direct path-that is, optimal (beta) motion. As the flashes are further enough apart in time, the impression of AM will deviate from the optimality, and finally, they will lose their continuity and be seen as two independent visual events, such that the first object goes out of existence and a second one is materialized at another location. On the other hand, if the absence of the object during the long ISI can be attributed to occlusion by the semicircular tube, amodal representation of a fully hidden object may bridge the spatiotemporal gap between the tokens via the path behind the curved occluder, resulting in the percept of curved motion.

Hence, we predicted that within the range of ISIs favorable for standard straight AM, curved motion is correspondingly less likely to be perceived but that, at long ISIs in which the default straight-path motion is weakened, the visual system increasingly favors the longer path behind the curved occluder, so as to ensure invisible but persisting object representation, which is otherwise not plausible.

The present study comprises a series of experiments that investigate the possibility of curved-path AM induced by amodal completion of the path. We examined this as a function of spatiotemporal parameters in Experiment 1. We measured the effects of token aspect ratio and the spatial gap between tokens and the semicircular occluder on the likelihood of curved-path motion percept in Experiments 2 and 3. In Experiment 4, we tested an alternative explanation for the curved motion effect that curved-path motion is simply due to priming of the curved path by the shape of the semicircular tube (Shepard \& Zare, 1983), rather than occlusion of the trajectory, using two stereoscopic displays, either compatible or incompatible with occlusion of the motion trajectory.

\section{General method}

\section{Participants}

Fifty-nine Rutgers University undergraduates took part in one of four experiments for course credit. There were 14 participants in Experiment 1, 20 participants in Experiment 2, 17 participants in Experiment 3, and 8 participants in Experiment 4. All had normal or corrected-to-normal vision and were naïve as to the purpose of the experiment.

\section{Apparatus}

In Experiments 1-3, stimuli were presented on a 17 -in. screen of $1,152 \times 864$ pixel (horizontal by vertical) resolution and a vertical refresh rate of $80 \mathrm{~Hz}$ of an Apple Power PC-G4/1.25 GHz eMac. In Experiment 4, stimuli were generated on a Macintosh G5 computer and were presented on a Mitsubishi Diamond Pro 2060u 21-in. monitor with a resolution of $1,024 \times 768$ pixels at a refresh rate of $120 \mathrm{~Hz}$. In Experiment 4, two stereo images were drawn to each eye on alternating frames (at $60 \mathrm{~Hz}$ ), with the use of CrystalEyes LCD shutter glasses and an E-2 infrared emitter (Stereographics Corporation). The experiments were programmed in MATLAB using the Psychophysics Toolbox extensions (Brainard, 1997; Pelli, 1997). The participants were seated in a dark room. In Experiments 
1-3, they viewed the display from a distance of approximately $60 \mathrm{~cm}$ without head restraint. In Experiment 4, the viewing distance was $100 \mathrm{~cm}$, and head position was stabilized with a chinrest.

\section{Stimuli}

Figure 1a shows the schematic illustration of the stimulus display. Stimuli were computer-generated AM sequences of two alternatively presented token frames, each of which was separated in time by the ISI. A token frame consisted of a small red/white textured rectangle $\left(1.00^{\circ} \times 0.61^{\circ}\right.$ in Experiments 1 and 3 and $0.8^{\circ} \times 0.45^{\circ}$ in Experiment 4; the aspect ratio was varied at nine levels in Experiment 2) on top of the center of each end of a semicircular tube with radially white-to-black-gradient color against black/white random-dot-patterned background. The width of the semicircular tube was $2.2^{\circ}$ in Experiments $1-3$ and $1.8^{\circ}$ in Experiment 4. The diameter of the tube, which corresponds to the spatial separation between the centers of alternately presented tokens, was manipulated in three levels (small, $3.73^{\circ}$; medium, $5.73^{\circ}$; large, $8.79^{\circ}$ ) in Experiment 1, to examine how spatial separation interacts with temporal separation (ISI). These levels were adapted from the range of spatial separation used in McBeath and Shepard's (1989) study, where they measured the deviation of AM from the straight path using motion tokens of different orientations. The diameter of the tube was fixed at $5.73^{\circ}$ in Experiments 2 and 3 and at $4.5^{\circ}$ in Experiment 4. During the ISIs, only the semicircular tube was presented against the background, without the rectangular tokens. Hence, the semicircular tube remained onscreen during the whole motion sequence. The duration of ISI varied at four levels $(100,233,367$, and $500 \mathrm{~ms}$ ) in Experiments 1, 2, and 4 and at three levels $(100,250$, and $400 \mathrm{~ms})$ in Experiment 3. The duration of a token frame varied at three levels $(60,120$, and $180 \mathrm{~ms})$ in Experiment 1 but was fixed at $150 \mathrm{~ms}$ in Experiments 2-4. Hence, the shortest SOA, which is the sum of token duration and ISI, was $160 \mathrm{~ms}$ (60-ms token duration and $100-\mathrm{ms}$ ISI), and the longest SOA was $680 \mathrm{~ms}$ (180-ms token duration and 500-ms ISI), such that the SOA values were comparable to the range of SOAs that previous curved AM studies used (e.g., Mcbeath \& Shepard, 1989; Shiffrar \& Freyd, 1990). By manipulating both ISI and token duration, we sought to determine whether ISI or SOA is more closely related to the percept of curved-path motion. On half of the trials, motion sequences started from a frame where a motion token appeared on the left end of the tube (the first frame in Fig. 1a), and on the other half, it started from the frame with a motion token on the right end of the tube (the third frame in Fig. 1a). On each trial, three and one half cycles of the motion sequence above were presented. QuickTime animations of motion sequences used in the present study are available at http:// rci.rutgers.edu/ $\sim$ sungho $4 /$ curvedAM.htm.

Procedure

Each trial started with a fixation cross presented at the center of the screen for $500 \mathrm{~ms}$, which was followed by three and one half cycles of an animation sequence like that shown in Fig. 1a. After the animation finished, the whole display disappeared, and participants were asked to determine whether the motion tokens appeared to be moving along the straight path between the positions of the two tokens or along a curved path behind (or through) the semicircular tube (see Fig. 1b). In particular, when the motion impression was ambiguous, they were instructed to select the straight path if tokens appeared to move in horizontal direction and the curved path if they appeared to move in vertical direction at their onsets and offsets. The participants were to press the " $\mathrm{v}$ " key to indicate that they perceived a straight-path motion and " $\mathrm{c}$ " to indicate that they perceived a curved-path motion. To assess how strongly and clearly they perceived AM, the participants were asked to rate the quality of the motion they perceived on a 3-point scale after the response regarding the motion path had been made. A rating of 3 represented a smooth continuous motion with a well-defined path; a rating of 1 represented a jerky or weak motion with a poorly defined path, or no motion; and a rating of 2 indicated a motion of intermediate motion quality and path definition. In cases where participants did not get any impression of AM but got the impression of two blinking objects in succession, they were asked to choose the straight path with a rating of motion quality of 1 .

\section{Analysis}

The main dependent measure was the proportion of curvedpath responses, with a secondary analysis of the motion quality rating. The results of motion quality rating were reported in Experiments 1 and 4 but were not included in Experiments 2 and 3, where the basic pattern of the findings was similar to that in Experiment 1. The data from 3 participants, 1 for each of Experiments 1-3, were excluded from the analysis, since they did not report any curved motion or did so only on a few trials.

\section{Experiment 1}

Experiment 1 examined whether the perceived path of AM can be biased from the shortest possible path toward a 
longer curved one by the presence of a curved occluder. We manipulated spatiotemporal parameters known to affect the quality and possibility of AM, such as ISI, token duration, and spatial separation, with primary focus on the effect of ISI on path choice. We expected the default straight-path motion to be dominant at short ISIs, because in the range of token duration (60$180 \mathrm{~ms}$ ) and ISI (100-500 ms) used in Experiment 1, the quality of (default straight-path) AM is known to be maximum at short ISIs and generally decreases with increasing ISI (Ekroll, Faul, \& Golz, 2008; Kahneman \& Wolman, 1970; Kolers, 1972). At long ISIs less conducive to a straight-path motion, however, we hypothesized that participants would be more liable to perceive motion along the longer, curved path through the semicircular tube, since occlusion of the motion trajectory behind the tube might account for the objective invisibility of the tokens during the long ISI.

\section{Method}

The combination of four ISIs, three token durations, and three token distances (semicircular tube diameters) yielded 36 different motion sequences. With ten repetitions of each sequence (five times leftward and five times rightward initial motion), each participant completed a total of 360 experimental trials, presented in random order, over four sessions. Each participant underwent 26 practice trials, followed by four sessions of experimental trials.

\section{Results}

Motion path analysis The results of Experiment 1 are presented in Fig. 2a. A repeated measures analysis of variance (ANOVA) was used to determine whether the perceived path of AM varied as a function of ISI, token duration, and token distance. The main effect of ISI was significant, $F(3,36)=96.96, p<.0001$, such that as the ISI became longer, the proportion of curved-path responses increased. However, the ANOVA did not yield any significant effects of either token duration or token distance, $F(2,24)=2.22, p>.10$, and $F(2,24)=1.41, p>.10$, respectively. No two-way or three-way interactions reached a significant level $(p s>.05)$.

Motion quality analysis The motion quality ratings were analyzed on the basis of the motion path participants chose over four ISIs. Figure $2 b$ summarizes how the AM quality depended on the perceived path of motion. Neither ISI nor path choice had a significant effect on motion quality rating, $F(3,36)=1.78, p=.17$, and $F(1,12)=1.06, p=$ .32 , respectively. The mean rating scores were 2.02 for straight and 2.09 for curved motion cases. However, the interaction between ISI and perceived motion path was significant, $F(3,36)=19.01, p<.001$. As ISI increased, the quality of straight-path motion sharply dropped, but that of curved motion showed an increasing trend. Separate ANOVAs for each motion path response confirmed that for straight motion cases, the effect of ISI on motion quality was significant, $F(3,36)=16.82, p<.001$, such that motion quality decreased as ISI increased, but that for curved motion, the effect of ISI was not significant, $F(3$, 36) $=1.44, p=.25$.

\section{Discussion}

Experiment 1 demonstrates that a curved occluder can induce a curved AM, which deviates from the default straight path. In particular, the results showed the systematic effect of ISI on the perceived motion path. At short ISIs, observers tended to perceive the default straight-path motion over a curved motion. However, as the ISI became longer, they were increasingly more likely to report a
Fig. 2 Results of Experiment 1. a Proportion of curved-path motion responses for the three token durations over the four Interstimulus intervals (ISIs). b Motion quality rating for straight- and curved-path motion over the four ISIs. Error bars represent \pm 1 standard error a

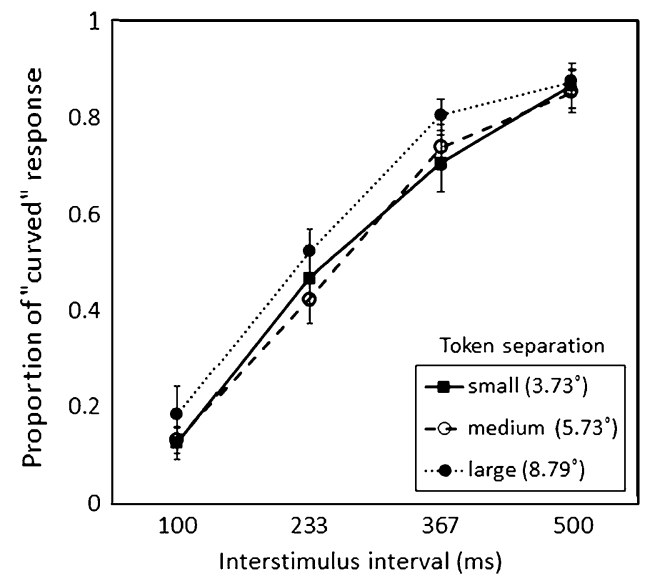

b

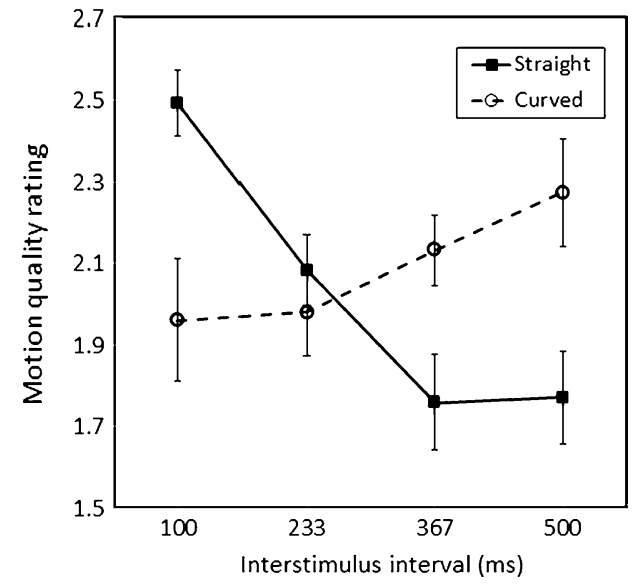


curved-path motion through the tube, rising above $50 \%$ at the longest ISIs.

Without the curved occluder (i.e., in a standard two-flash AM display), long ISIs would have resulted in simply less convincing and weaker motion or the percept of two discrete objects in succession without any motion. Then, why does the presence of the semicircular occluder lead to curved-path AM at long ISIs? We speculated that this relates to minimization of unexplained invisibility (cf. Scherzer \& Ekroll, 2009). As the ISI becomes longer, the amount of unexplained absence of the tokens in space-time, which corresponds to the volume in the three-dimensional (3-D) space-time diagram (see Fig. 1c) consisting of two spatial dimensions and a third time dimension (e.g., Adelson \& Bergen, 1985), increases for the straight motion interpretation. This increase in unexplained absence of the tokens might result in a decrease in motion strength, as suggested by decreasing motion quality rating with increasing ISI. On the other hand, this is not the case for the curved motion interpretation, because the invisibility of the tokens during the ISI can be explained by the occluder, irrespective of ISI. That is, once a sudden offset of a motion token is attributed to occlusion, amodal completion of motion trajectory behind the occluder ensures the persistence of a moving object via a curved motion trajectory.

This interpretation is supported by a recent study by Scherzer and Ekroll (2009), who demonstrated that invisible representations of objects during the ISI contribute to enhance the quality of AM. They found that at long ISIs (over $300 \mathrm{~ms}$ ), which is a comparable range of ISIs favoring curved motion in the present experiment, the motion appeared smoother when the background was masked during the ISI than when the background remained unchanged. The smoothing effect cannot be explained by low-level motion-energy types of models such as the Reichardt model (Reichardt, 1961), since it occurred at relatively long ISIs that are beyond the temporal resolution for low-level motion detectors to integrate motion signals. They concluded that if the absence of the motion token during the ISI is attributed to a sudden appearance of a mask screening the entire frame, rather than the token itself, the object can be perceived to come into and go out of sight while continuing to exist behind the mask, resulting in enhancement of the perceptual quality of AM.

The lack of significant effects of token duration and spatial separation suggests that long temporal separation is the critical cue to induce the percept of curved-path motion, such that the visual system interprets the prolonged absence of a token as occlusion by the semicircular tube, regardless of levels of other spatiotemporal parameters. The null effect of spatial separation might be explained by invariance of the ratio between the lengths of straight and curved paths across three levels of token separation. As token separation increases, the amounts of unexplained absence for both the straight motion path and the curved path increase together in the same proportion. The null effect of token duration is consistent with Scherzer and Ekroll's (2009) finding that the enhanced smoothness of AM was observed over a range of token duration (47-424 ms), except for the brief duration (47 ms). On the other hand, in the AM literature, it is known that, generally, SOA is the most critical temporal determinant of AM (i.e., onset-onset rule; Kahneman, 1967; Kolers, 1972) and that the quality of AM is little affected by how a given SOA interval is distributed between token duration and ISI. In order to investigate the effect of SOA on path choice and motion quality, we plotted the results of Experiment 1 pooled across all participants against SOA in Fig. 3. As can be expected from the null effect of token duration, sharp increases in the proportion of curved-path responses happened mostly between the points where the ISI increased, but within each level of ISI, the modulation of motion percept by SOA was less salient (Fig. 3a). On the other hand, Fig. 3b showed that the quality of standard straight-path motion decreased quite linearly with increasing SOA. This suggests that even though the temporal separation between motion tokens is critical for the percept of longer, curved motion via amodal completion, the quality of straight-path motion is still dependent on SOA, as the AM literature has suggested. However, we cannot exclude the possibility that the relative contribution of token duration to the SOA's effect on path choice could have been less salient than that of ISI, since we used a narrower range of token durations, as compared with that of ISIs.

An alternative explanation of the influence of ISI on path choice might be that completion of a longer (curved) path requires longer temporal separation, generalized by Korte's (1915) third law. However, without the semicircular tube, it is unlikely for AM to deviate from the straight path even at long ISIs. Another related, more plausible explanation is priming of curved path by the shape of the semicircular tube, not involving occlusion of the trajectory (Shepard \& Zare, 1983). Experiment 4 examined the contribution of path priming using two stereoscopic displays, either compatible or incompatible with occlusion of the motion trajectory.

\section{Experiment 2}

The aim of Experiment 2 was to test how the curved AM we observed in Experiment 1 is affected by the shape of motion tokens. We employed nine rectangular shapes as motion tokens, by manipulating their height and width separately at three levels, as in Fig. 4. According to Korte's (1915) third law, the optimal path of AM is determined solely on the basis of spatiotemporal separations between 
Fig. 3 Results of Experiment 1 plotted on data pooled across all participants against stimulus onset asymmetry $(\mathrm{SOA}=$ token duration + interstimulus interval [ISI]). a Proportion of curved-path motion responses. b Motion quality rating for straight- and curved-path motion. Error bars represent \pm 1 standard error of the mean of the pooled data a

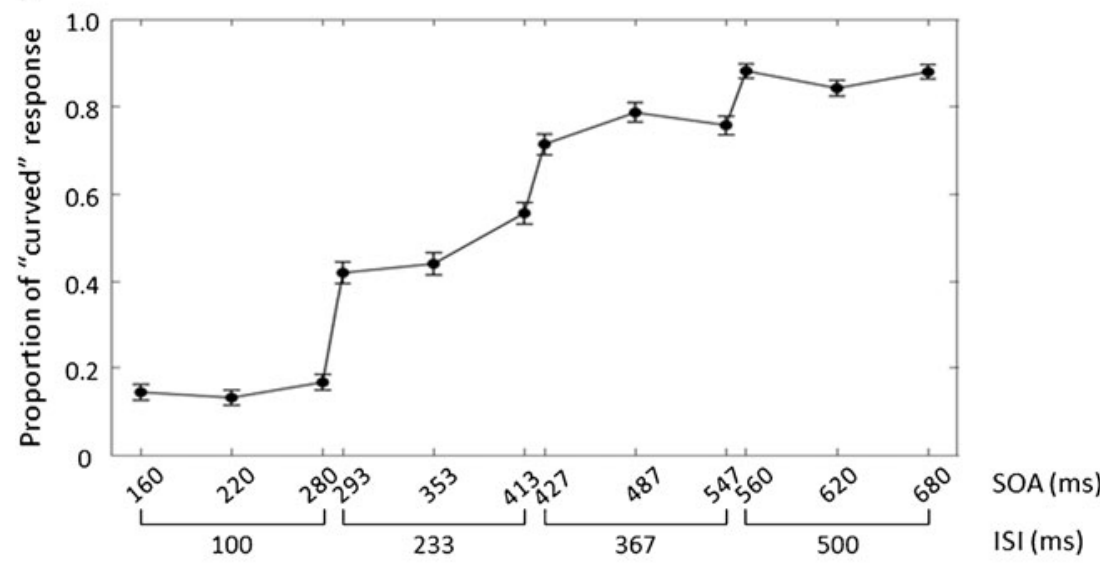

b

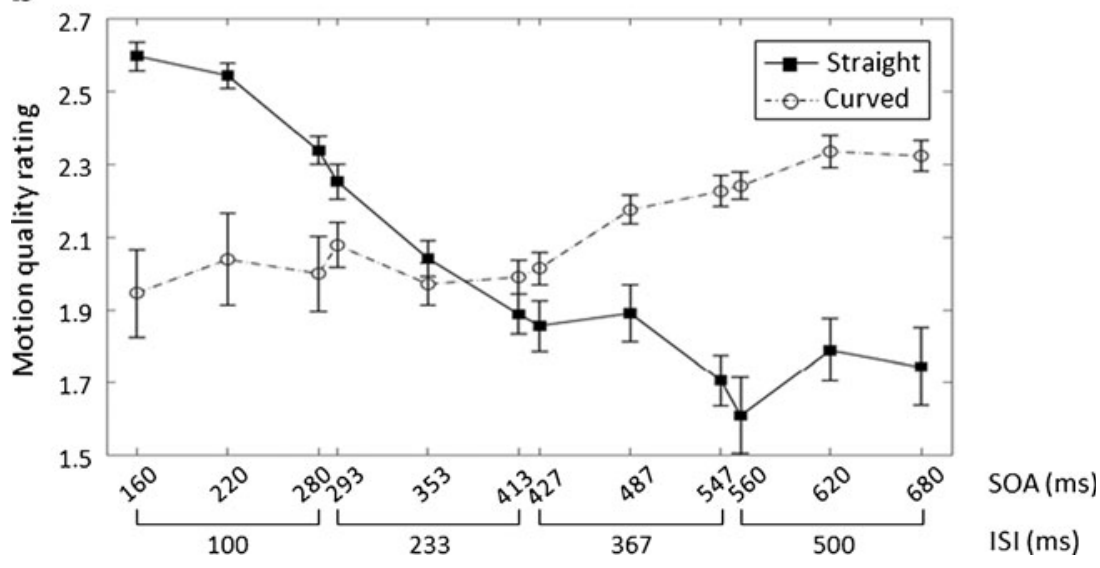

motion tokens. On this view, other figural factors, such as shape, symmetry, or orientation, should not affect the motion path. Hence, any influence of token shape on the curved-motion percept should be explained by alternative processes than simple spatiotemporal constraints. In particular, our interest was in the comparison between tokens with the same area but different orientations, such as tokens

Fig. 4 a Nine tokens used in Experiment 2. They were generated by the combination of three levels of token width and height. Levels 1, 2, and 3 represent one, two, and threefifths of semicircular tube width. b Results of Experiment 2: Proportion of curved-path motion responses for the three token heights over three widths. Error bars represent \pm 1 standard error a

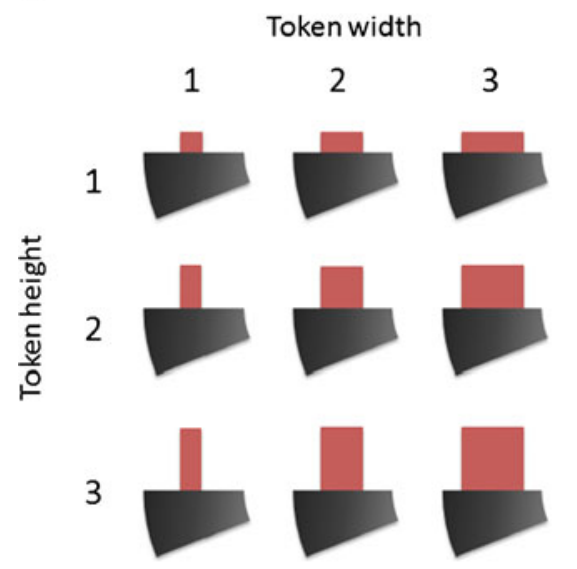

in cells $(1,2)$ and $(2,1),(1,3)$ and $(3,1)$, and $(2,3)$ and $(3$, 2) in Fig. 4a.

\section{Method}

We manipulated token heights and widths at three levels, respectively, such that tokens could be one fifth, two fifths, b

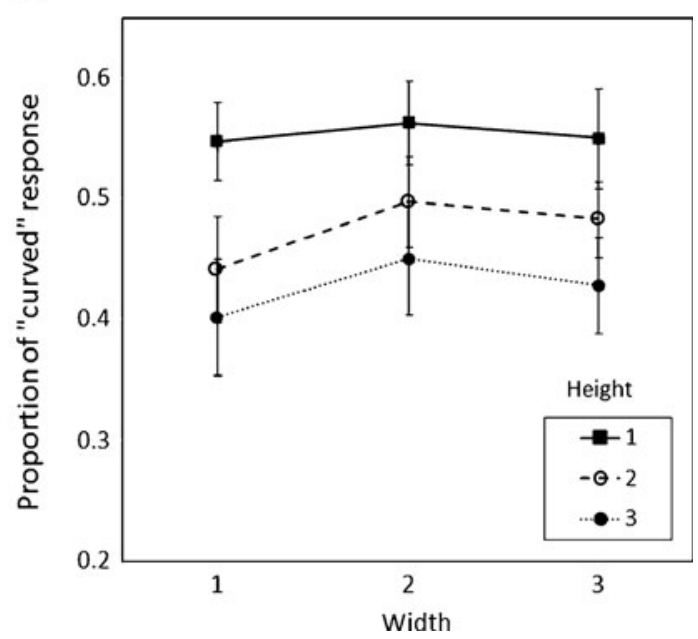


and three fifths of tube width $\left(2.2^{\circ}\right)$ in both height and width (Fig. 4a). By combination of the levels of the two dimensions, nine tokens were constructed. The diagonal length of the largest token $\left(1.87^{\circ}\right)$ was smaller than the tube width, so physically all the tokens could be fully hidden behind the tube. The combination of four ISIs, three token heights, and three token widths yielded 36 different motion sequences. With ten repetitions of each sequence, each participant completed a total of 360 experimental trials interleaved, presented in random order, over four sessions.

\section{Results}

The results of Experiment 2 are presented in Fig. 4b. To determine whether the percept of curved-path motion via the semicircular tube was affected by token aspect ratio, a $4 \times 3 \times 3$ repeated measures ANOVA was conducted on the factors ISI, token height, and token width. The main effect of ISI was significant, as in Experiment 1, $F(3,54)=49.36, p<$ .0001 , such that the proportion of curved-path responses increased with increasing ISI. The main effect of token height was also significant, $F(2,36)=5.84, p=.006$. Curvedpath responses increased monotonically with decreasing token height. But the effect of token width was not significant, $F<1$. A token height $\times$ ISI interaction was marginally significant, $F(6,108)=1.93, p=.08$, such that the difference among levels of token height was bigger with increasing ISI. No other two-way or three-way interactions reached a significant level, $p \mathrm{~s}>.05$. Especially, horizontally oriented tokens produced higher curved-path motion response than did vertically oriented tokens in all three same-area pairs, as shown in Fig. 3b. Planned paired $t$-tests conducted for the three pairs of horizontal versus vertical tokens showed that the advantage of horizontally over vertically oriented tokens was significant at the small-size pair, $t(18)=2.16, p=.044$, marginally significant at the medium-size pair, $t(18)=1.88$, $p=.078$, but not significant at the large-size pair, $t(18)=$ $0.584, p=.567$.

\section{Discussion}

The results showed that the shorter the tokens were in height, the more likely participants were to perceive the curved-path motion but that token width hardly affected motion path. For tokens of the same area, horizontally oriented ones were more likely to be perceived as moving along a curved path through the semicircular occluder than were vertically oriented ones. For curved-path motion, a token should move vertically up and down behind the occluder at its onset and offset. Hence, the effect of token orientation suggests that motion in the vertical direction was more likely to be seen for horizontally oriented tokens than for vertically oriented tokens, whereas horizontal motion is more compatible with vertically oriented tokens. Considering that horizontally oriented tokens (i.e., their primary axes) are co-aligned with straight path but orthogonal to curved path (and vice versa for vertically oriented tokens), this finding is quite contradictory to the previous studies showing dominance of AM co-aligned with object orientation over orthogonal (Werkhoven, Snipe, \& Koenderink, 1990).

Preference for curved-path motion percept with horizontally oriented tokens seems to call for a surface-based explanation (e.g., Nakayama, He, \& Shimojo, 1995), rather than low-level spatiotemporal motion integration. Whenever a surface is moving in front of or behind another surface, the occluded surface entails progressive deletion of texture (or decrease in image size) at the border where the two surfaces meet and also can be reversibly disoccluded with entailing progressive accretion (or increase in image size) in this time (Gibson, Kaplan, Reynolds, \& Wheeler, 1969; Michotte et al., 1964/1991). Several studies have corroborated that accretion and deletion cues are critical for the percept of kinetic occlusion (Andersen \& Cortese, 1989; Kaplan, 1969; Scholl \& Pylyshyn, 1999; Yonas, Craton, \& Thompson, 1987). Recall that our AM sequences do not involve progressive occlusion/disocclusion (accretion/deletion) cues; objects simply appear or disappear without intermediate steps of size change. However, a less tall token undergoes a smaller change in height at its offset or onset than does a taller token, creating a display sequence that is more comparable to progressive accretion/ deletion. Such tokens might, consequently, be more likely to be perceived as being progressively occluded (and disoccluded) by the semicircular occluder. In other words, the smaller the tokens are in height, the more readily their disappearance can be attributed to being hidden behind the occluder and, thus, moving along the curved path. It follows from this analysis that the difference between horizontally and vertically oriented tokens might result solely from the effect of token height and that there is no unique effect of target orientation other than that of token height: For a curved-path motion percept, a horizontally oriented token, being less tall in the first place, undergoes a smaller change in height at offset or onset than does a vertically oriented token of the same size.

\section{Experiment 3}

In Experiment 3, we introduced a spatial separation between the tokens and the occluder in order to study the influence of static amodal completion on path choice. In AM displays shown in Experiments 1 and 2, the rectangular tokens appear on top of the ends of the semicircular occluder, allowing them to be perceived as partially 
occluded behind the occluder. The amodally completed surface representation might be important for the percept of curved-path motion, since it implies that the token is already partly behind the occluder and, thus, shifts the token's center of mass toward the occluder (Shimojo \& Nakayama, 1990). The aim of Experiment 3 was to explore the contribution of static amodal completion to curved-path motion by introducing a gap between the occluder and the token, breaking the T-junctions that served as occlusion cues. When the token no longer abuts the occluder, static amodal completion of motion tokens behind the occluder should be diminished. However, Anstis and Ramachandran (1985) and Gerbino (1984) have shown that even when objects do not abut directly, the disappearance of an object near another object can generate the impression of occlusion. If static amodal completion of motion tokens cued by T-junctions is critical for curved-path motion through the semicircular occluder, the tendency toward curved-path motion would disappear as soon as a small gap is introduced between motion tokens and the occluder. If the kinetic structure of moving objects itself is critical, curved-path percepts would be sustained even when the static occlusion cues (T-junctions) are removed.

\section{Method}

We introduced spatial gap between motion tokens and tube ends (see shapes below the $x$-axis in Fig. 5). There

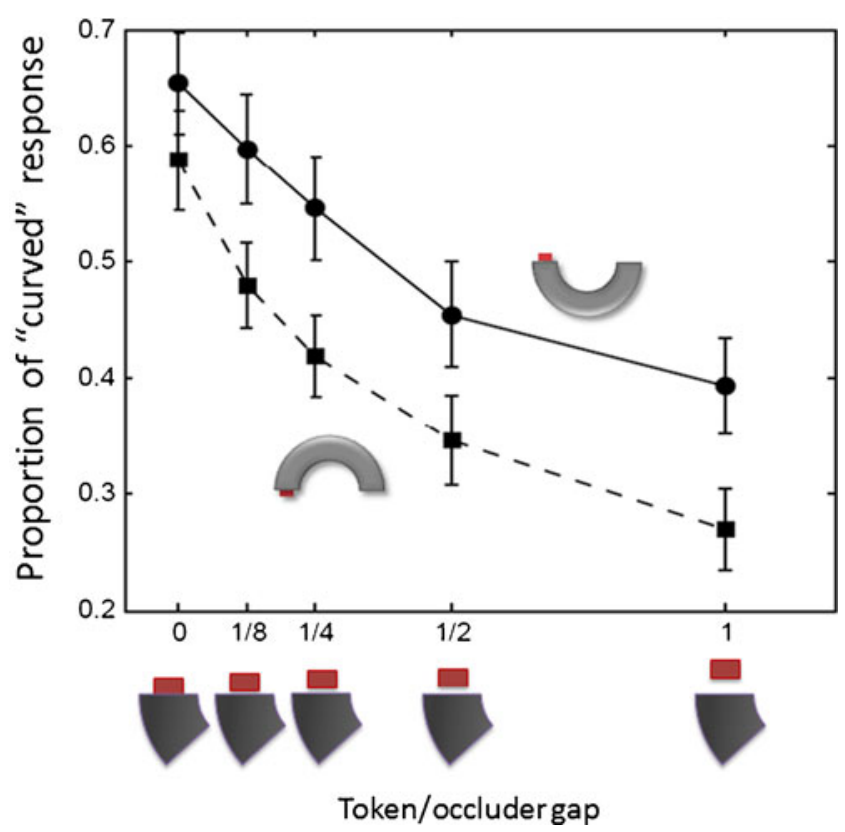

Fig. 5 Results of Experiment 3: Proportion of curved-path motion responses for upright and upside-down displays each over five gaps between tokens and an occluder. Error bars represent \pm 1 standard error were five levels of gap, including a zero-gap case, where displays were the same as those used in Experiment 1, and four levels of nonzero gaps, which were one eighth, one forth, and one half of token height and one of token height. On half of the trials, the displays were upright, but on the other half, the displays were rotated $180^{\circ}$ (upsidedown). The combination of three ISIs, five token gaps, and two display orientations (upright and upside-down) yielded 30 different motion sequences. With ten repetitions of each sequence, each participant completed a total of 300 experimental trials interleaved, presented in random order, over four sessions.

\section{Results}

The results of Experiment 3 are presented in Fig. 5. To determine whether curved motion through a semicircular tube is affected by token gap and display orientation, a $3 \times$ $5 \times 2$ repeated measures ANOVA was conducted on factors of ISI, token gap, and display orientation. The main effect of ISI was significant, as in Experiment $1, F(2,30)=$ $32.84, p<.0001$. The effect of token gap was also significant, $F(4,60)=11.57, p<.001$, such that the proportion of curved-path responses decreased monotonically with increasing token gap. Planned paired $t$-tests showed that every level of gap size was significantly different from each other, $p$ s $<.024$, except for between the two largest gaps, $p=.12$. ANOVA also revealed a significantly more curved-path responses for upright displays $(M=.53)$ over upside-down displays $(M=.42)$, $F(1,15)=6.24, p=.025$. No two-way or three-way interactions reached significance, $p \mathrm{~s}>.05$.

\section{Discussion}

The main finding in this experiment was that curved-path responses decreased monotonically with increasing token gap, with an approximately constant advantage for upright over upside-down displays. The decrease in curved-path motion responses started as soon as the smallest gap (one eighth of token height) was introduced, but even without Tjunctions, curved-path motion was still persistent. The rate of drop in curved-path responses was highest at the smallest gap, but it was not very different from those at larger gaps, except for that at the largest one. These results suggest that static amodal completion of motion tokens alone cannot explain curved-path motion, even though it contributes to such percept. The length of the straight path between two tokens is constant irrespective of the gap size. However, for curved-path motion, the objective invisibility of tokens (before entering and after coming out of the tube), which is not explained by occlusion, increases with the gap size, resulting in a drop in curved-path motion percept. This 
result, along with the effect of token height observed in Experiment 2, suggests that proximity between centers of mass of tokens and nearby larger surface is a critical spatial cue for illusory occlusion of motion trajectory, at least when the temporal separation between token frames is long enough.

The preference for valley-shaped over hill-shaped path orientations might be attributed to the presence of an implied gravitational frame. When the semicircular tube is below the motion tokens, a physical token would be able to fall freely down to the lowest point, simply due to the effect of gravity, and then (assuming no dissipative forces in the system) would have enough kinetic energy to regain its original height at the other end of the tube. In contrast, when the tube lies above the tokens, an external force would be required to overcome gravity to initiate the motion. Previous researchers have suggested that dynamic object representations might be constrained by fundamental ecological and physical principles (Freyd, 1987; Shepard, 1984). The effect of perceived gravity on motion has been reported in the studies of so-called representational momentum: the mislocalization of an object in the direction of its implied motion after it disappears (Freyd \& Finke, 1984). A number of studies have demonstrated that a stimulus moving downward produced a larger forward memory displacement than did a stimulus moving upward (Hubbard, 2001; Nagai, Kazai, \& Yagi, 2002) and that even horizontally moving and stationary stimuli could produce downward memory displacement (Bertamini, 1993; Freyd, Pantzer, \& Cheng, 1988; Hubbard \& Ruppel, 2000; Reed \& Vinson, 1996). On the other hand, it is known that attentional resolution is higher in the lower visual field than in the upper visual field (He, Cavanagh, \& Intriligator, 1997). Hence, the advantage of the valley-shaped occluder for curved-path motion might be alternatively explained by an attentional advantage for the lower visual field. Further studies are required to investigate the role of implied gravity.

\section{Experiment 4}

One possible explanation of the curved AM observed in the previous experiments is that it was simply due to path priming (Shepard \& Zare, 1983); that is, the mere presence of the semicircular tube primed participants to perceive curved motion. Shepard and Zare demonstrated that when a gray curved path was briefly flashed in the space between two alternating stimuli, AM appeared to follow the gray path rather than the most direct route. The minimum ISI necessary to produce AM following the gray arc path increased linearly with the length of the indirect, curved path, consistent with Korte's (1915) third law. Apparently, the role of the semicircular tube we used is similar to Shepard and Zare's gray arc path, since both of them appear to guide motion trajectory. To distinguish the current curved-path motion effect from path-guided AM, Experiment 4 used two 3-D stereoscopic displays, either compatible or incompatible with occlusion of the motion trajectory. In a tube-in-front display, we placed the semicircular tube in front of the motion tokens in stereoscopic depth, providing an opportunity for the tokens to move amodally behind the semicircular occluder. In a token-front display, the semicircular tube was presented in back of the motion tokens, which would not allow occlusion of the motion trajectory by the tube. However, monocular occlusion cues of T-junctions, formed by vertical edges of tokens and horizontal edges of tube ends, could still give observers the impression of tokens occluded by the tube in spite of binocular disparity cues indicating tokens in front. To prevent such a conflict in depth defined by binocular disparity and by T-junctions, we displaced tokens slightly lower toward the tube than in the tube-in-front display (see Fig. 6b). In this configuration, both binocular disparity and the T-junctions were consistent with the percept of tokens as in front. If the curved-path motion we observed in Experiments 1-3 was due to occlusion of the motion trajectory, curved motion should not be perceived in the token-in-front display. However, if path priming is a good explanation for curved-path motion, 3-D depth manipulation should not change the percept of curvedpath motion.

\section{Method}

A gray rectangular frame with a rectangular aperture $\left(13.5^{\circ} \times 9.1^{\circ}\right)$ was stereoscopically presented at zero binocular disparity (in the monitor plane). The background, motion tokens, and the semicircular tube were seen through the aperture. The background and motion tokens were always placed in depth planes of 24 pixel (approximately $32 \mathrm{arcmin}$ ) and 13 pixel (approximately 17.5 arcmin) disparities, respectively. We manipulated the stereoscopic depth of the semicircular tube in two levels: 11 pixel disparity either in front or behind the motion tokens (Figs. 6a and 6b). In the token-in-front display, the motion tokens were displaced downward by $9 \operatorname{arcmin}(1 / 3$ of token height), partially occluding the ends of the semicircular tube. The combination of two surface depth orders and four ISIs yielded eight different motion sequences. With 16 repetitions of each sequence, each participant completed a total of 128 experimental trials interleaved, presented in random order, over four sessions, following 26 practice trials.

Before the experiment, participants were tested for their stereo vision by viewing two figures presented in different 
Fig. 6 Illustration of stimuli used in Experiment 4: Tube-in-front display (a) and token-in-front display (b). Shadows are presented for illustrating stereoscopic surface depths specified by binocular disparities and was not included in the actual stimuli. c Results of Experiment 4: Proportion of curved-path motion responses for tube-in-front and token-in-front displays over the four interstimulus intervals. Error bars represent \pm 1 standard error

\section{a}

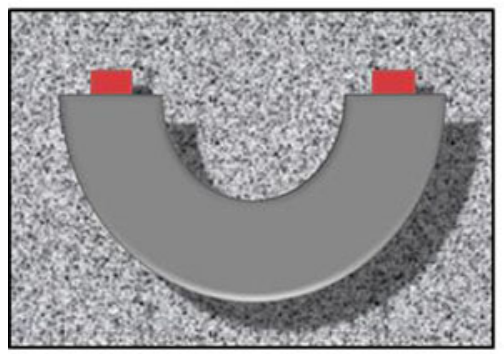

b

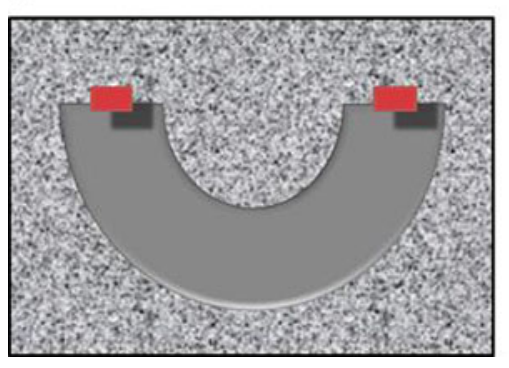

C

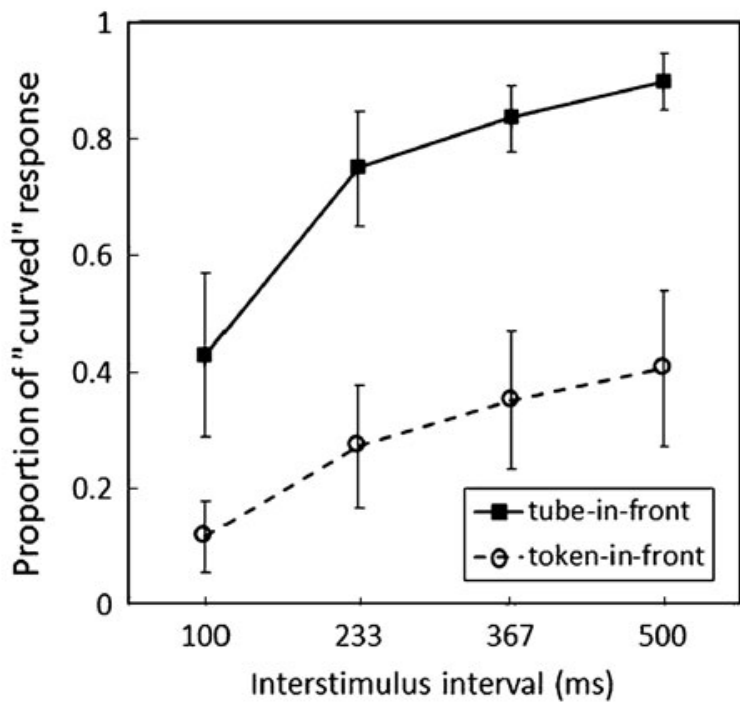

depths via the LCD shutter glasses and adjusting the depth of a figure to match to that of another. All of them accurately adjusted relative depth. Each trial started with a fixation cross presented at the center of the background for $600 \mathrm{~ms}$, and then a semicircular tube appeared for $600 \mathrm{~ms}$ to provide sufficient time for perceiving the stereoscopic depth, which was followed by three and one half cycles of an animation sequence like that shown in Fig. 1a.

\section{Results}

The results of Experiment 4 are presented in Fig. 6b. A two-way repeated measures ANOVA on surface order (token-in-front vs. tube-in-front) and ISI revealed that the main effect of surface order was significant, $F(1,7)=$ $10.42, p=.014$. The proportion of curved path responses was much higher in the tube-in-front display $(M=72.9 \%)$ than in the token-in-front display $(M=28.7 \%)$. The main effect of ISI was also significant, as in the previous experiments, $F(3,21)=10.69, p<.001$. However, a surface order $\times$ ISI interaction was not significant, $F(3,21)=$ $1.29, p=.305$.

The motion quality rating was analyzed in a threeway ANOVA on path choice (curved vs. straight) over four ISIs in two surface orders (see Fig. 7). Since some participants exclusively chose one motion path in some conditions, a within-subjects analysis was impossible, so we pooled the motion quality rating data across participants. Motion quality rating was significantly higher for the tube-in-front displays $(M=2.33, S D=0.74)$ than for the token-in-front displays $(M=1.89, S D=0.78), F(1$,
$1008)=47.14, p<.001$, and also it decreased with increasing ISI, $F(3,1008)=47.14, p<.001$. All two- and three-way interactions involving ISI (ISI $\times$ depth order, ISI $\times$ path choice, and ISI $\times$ depth order $\times$ path choice) were significant, $F(1,1008)=13.76, p<.001, F(1,1008)=$ $20.80, p<.001$, and $F(3,1008)=4.08, p=.007$, respectively. No other main effects or interactions were significant. Separate ANOVAs for each path choice showed that the quality of straight motion dropped more sharply with increasing ISI in the token-in-front displays than in the tubein-front displays, $F(3,496)=4.75, p=.003$. On the other hand, the quality of curved motion was enhanced with increasing ISI in the tube-in-front displays but was not modulated by ISI in the token-in-front displays, $F(3,512)=$ $9.22, p<.001$.

\section{Discussion}

The higher proportion of curved-motion responses in the tube-in-front than in the token-in-front displays supports the idea that the present curved motion effect is due to the perceived occlusion of the motion trajectory, rather than mere priming of the curved path by the semicircular tubesimilar to the phenomenon of path-guided AM (Shepard \& Zare, 1983). Moreover, in the tube-in-front displays, both the probability and the quality of curved-path motion were much higher than in previous experiments where the occlusion of motion tokens was defined only by monocular depth cues (T-junctions). These results suggest that binocular depth cues for token occlusion strongly facilitate amodal completion of the motion trajectory behind a curved occluder. 
Fig. 7 Motion quality rating in Experiment 4 for tube-in-front and token-in-front displays over the four interstimulus intervals, on trials of the straight-path motion response (a) and curvedpath motion response (b) a

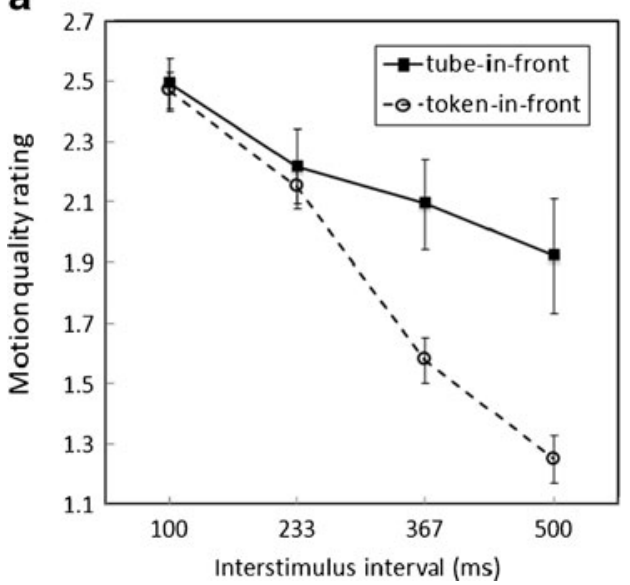

b

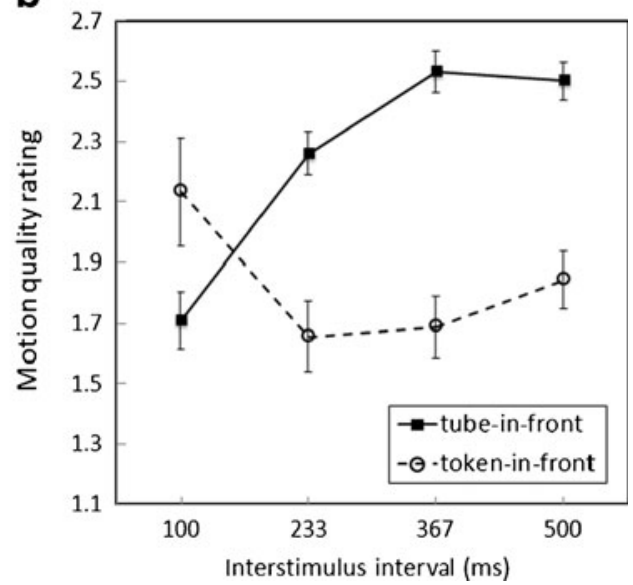

However, even in the token-in-front displays, which do not support occlusion of the motion trajectory, participants still chose curved-path motion on a nonnegligible proportion of trials. This suggests some role for path priming by the shape of the semicircular tube, similar to the path-guided AM (Shepard $\&$ Zare, 1983). However, the quality of curved motion was much worse in the token-in-front displays than in the tubein-front displays. Considering that the two surface depth orders were interleaved within the same block so that participants could directly compare them across trials, this difference is very likely to reflect differences in the strength of the motion signal generated by amodal completion of the motion trajectory versus by path priming. Hence, even if path priming can partly explain curved-path motion, its contribution to the curved-motion percept seems to be much smaller than that of amodal completion.

Interestingly, not only for curved- but also for straight-path motion, motion quality was much worse in the token-in-front than in the tube-in-front displays; at the longest ISI, the quality of straight motion $(M=1.25)$ generated by token-in-front displays was very close to 1 . We conjecture that in token-infront displays, it might have been more likely that long ISIs lead to the impression of two alternately "materializing" and "dematerializing" objects (with no percept of motion), because long disappearance of a motion token, previously shown "floating" in front of other surfaces, is easier to be interpreted as "evaporation." Even though the curved motion signal was weak in the token-in-front displays, our participants might have been biased to choose the curved path, since straight motion was even weaker.

\section{General discussion}

The present study investigated the possibility of curved AM induced by the occlusion of a moving object behind a curved occluder. The results confirm that curved paths can be induced by the presence of the curved occluder and show that the likelihood of such a percept was modulated by several temporal, as well as spatial, factors mediating the interpretation of occlusion of motion trajectory. A curved path was most likely with long ISIs, objects less tall and close to the occluder, U-shaped tube occluders, and binocular depth cues consistent with occlusion.

As a subjective measure, our task of motion path report has some limitations, including observer/experimenter bias and other cognitive influences. Participants' curved motion responses were generally more frequent than we had expected, on the basis of our own impressions, to the experimental displays, especially at an intermediate range of ISIs. Some participants informally reported that they were surprised by their own impression of curved motion after their sessions. Perhaps, once they perceived extraordinary but compelling curved motion at long ISIs, they could be reluctant to see the default straight motion even at relatively shorter ISIs (i.e., a "mental set" was created by the strongest stimuli). In addition, there was some interobserver variability worth noting. A few participants did not see curved motion at all throughout the whole experimental session. Bistable motion can be affected by observers' attention and intention (Kohler, Haddad, Singer, \& Muckli, 2008; Ramachandran \& Anstis, 1983; Wertheimer, 1912/1961). If observers' attention was strongly captured by the default straight path, it might have been difficult for them to flexibly switch their perceived trajectory to the alternative curved path. Further studies are required to more objectively measure the nature of curvedpath motion induced by kinetic occlusion, using indirect and objective tasks (e.g., Flombaum \& Scholl, 2006; Kawachi \& Gyoba, 2006; Yantis \& Nakama, 1998).

Physical plausibility of object occlusion

One issue possibly raised in the present study is the effect of apparent physical plausibility. Many vision researchers 
have suggested that fundamental principles of physics and regularities in the structure of the environment, such as object conservation, occlusion, surface rigidity, and so on, might be incorporated as constraints on early visual processing of motion perception (Freyd, 1987; Gibson, 1979; Marr, 1982; Shepard, 1984). Previous AM studies have demonstrated that the path of AM is influenced by the physical constraints of object permanence (Gerbino, 1984), occlusion (Anstis \& Ramachandran, 1985; Ramachandran et al., 1986), rigidity (Farrell \& Shepard, 1981; Kolers \& Pomerantz, 1971), and biomechanics (Shiffrar \& Freyd, 1990).

The present finding of the ISI effect on path choice suggests that the visual system tends to minimize the amount of unexplained invisibility of moving objects. The amount of unexplained absence of motion tokens increases with increases in ISI for the default straight motion interpretation, but it is essentially zero for curved-path motion interpretation no matter how long the ISI is, if the presence of curved occluder explains the absence of objects. Thus, if the tendency of increasing curved-path responses with ISI reflects this interpretation, this would mean that the visual systems is constrained to minimize the amount of unexplained absence, as well as the length of motion path (e.g., information minimization, Foster, 1975; Shepard, 1984; motion minimization, Farrell, 1983) and weigh them against each other when they are in conflict.

This analysis is closely related to the concept of object permanence. Object permanence is achieved when changes in the visual scene are understood in terms of stabilities in the distal world, using the regularities in the pattern of change to identify that which remains unchanged (Gibson, 1979; see also Feldman \& Tremoulet, 2006). An occluded surface entails progressive deletion of texture and also can be reversibly disoccluded with entailing progressive accretion in this time (Gibson et al., 1969). When these subtle visual cues indicate that objects have temporarily gone out of sight, the disappearances contribute significantly to the persistence of object representations during occlusion (Flombaum \& Scholl, 2006; Kawachi \& Gyoba, 2006; Scholl \& Pylyshyn, 1999). The advantage of less tall motion tokens for curved-path motion observed in Experiment 2 suggests that this regularity in the structure of optical transition entailing occlusion is incorporated for the perception of ambiguous AM displays.

Previous studies demonstrated that the visual system tends to avoid mysterious abrupt materializing and dematerializing of an object, which does manifest nonpermanence across space and time but, instead, preserve object persistence in space-time by generating illusory occlusion of objects if an occluder is available (Ramachadran et al., 1986; Sigman \& Rock, 1974). On the other hand, the present study suggests that the visual system can exploit spatiotemporal cues, such as ISI and the height of a motion token, to generate continuity of object representation behind an occluder, rather than resorting to problemsolving-like cognitive process, as Rock (1983) proposed (Ekroll et al., 2008).

The advantage of valley-shaped over hill-shaped paths can be understood as relating to gravitational forces, as explained above. Moreover, the "tube"-like appearance of our semicircular occluder might have facilitated the percept that the object was passing through it, which would also explain (as would ordinary occlusion) why the intervening motion is not visible. Therefore, it might be that the visual system tends to choose an interpretation that maximizes physical plausibility of occlusion, on the basis of spatiotemporal cues reflecting regularity in optical transition occurring at occlusion of moving objects.

\section{Occlusion and path priming}

In AM displays where two spatially separated objects are presented sequentially, the shortest possible path-in most cases, the straight path-is the default motion trajectory. However, deviations from straight AM have been reported in several other contexts (Anstis \& Ramachandran, 1986; Foster, 1975; Kolers \& Pomerantz, 1971; Shepard \& Zare, 1983; Shiffrar \& Freyd, 1990). As was mentioned earlier, among the curved motion phenomena reported in the AM literature, the case most comparable to the present study would be path-guided AM (Shepard \& Zare, 1983). Shepard and Zare found that when a gray arc band connecting two alternately displayed black dots is flashed during the ISI, a strong illusion is created of motion along the gray band, rather than the shorter linear path. This path priming effect is similar to the curved-path motion observed in the present study, in that both cases involve motion apparently "guided" by a visible curve. However, a critical difference between the curved gray band used in Shepard and Zare and the semicircular tube used in the present study is that the former is given only during part of the ISI, while the latter is shown over the whole motion sequence. Shepard and Zare hypothesized that the gray band was perceptually interpreted as a blur generated by the moving dot. In contrast, in our displays, nothing is flashed during the ISI; the occluder is static and always visible (before, during, and after the motion tokens). This difference, as well as the influence of token shape we found in Experiment 2, seems to preclude a similar low-level explanation. Furthermore, the effect of 3-D surface order on path choice strongly supports that the curved-path motion we found involves occlusion of motion trajectory, rather than just priming of curved path. While Shepard and Zare's displays can be thought of as inducing modal completion of the motion trajectory, 
ours apparently induce only amodal completion. Hence, while the two phenomena are similar, they are induced by different types of stimulus conditions and seem to tap qualitatively different mechanisms.

\section{Conclusion}

In conclusion, the present study suggests that the amodal representation of a fully occluded object can strongly modulate the trajectory of object motion by bridging the gap between two partially occluded tokens via a curved path, thereby permitting object permanence behind the curved occluder.

Acknowledgment We wish to thank Thomas Papathomas, Brian Scholl, Adriane Seiffert, and three anonymous reviewers for helpful comments. This work was supported in part by NIH (NEI) EY021494.

\section{References}

Adelson, E. H., \& Bergen, J. R. (1985). Spatiotemporal energy models for the perception of motion. Journal of the Optical Society of America A, Optics and Image Science, 2, 284-299.

Andersen, J. A., \& Cortese, J. M. (1989). 2-D contour perception resulting from kinetic occlusion. Perception \& Psychophysics, 46, 49-55.

Anderson, B., Singh, M., \& Fleming, R. (2002). The interpolation of object and surface structure. Cognitive Psychology, 44, 148-190.

Anstis, S., \& Ramachandran, V. S. (1985). Kinetic occlusion by apparent movement. Perception, 14, 145-149.

Anstis, S., \& Ramachandran, V. S. (1986). Entrained path deflection in apparent motion. Vision Research, 26, 1731-1739.

Bertamini, M. (1993). Memory for position and dynamic representations. Memory \& Cognition, 21, 449-457.

Blake, R., Ahlström, U., \& Alais, D. (1999). Perceptual priming to invisible motion. Psychological Science, 10, 145-150.

Brainard, D. H. (1997). The Psychophysics Toolbox. Spatial Vision, 10, 433-436.

Bruno, N., Bertamini, M., \& Domini, F. (1997). Amodal completion of partly occluded surfaces: Is there evidence for a mosaic stage? Journal of Experimental Psychology: Human Perception and Performance, 23, 1412-1426.

Burke, L. (1952). On the tunnel effect. Quarterly Journal of Experimental Psychology, 4, 121-138.

Clocksin, W. F. (1980). Perception of surface slant and edge labels from optical flow: A computational approach. Perception, 9, 253-269.

Ekroll, V., Faul, F., \& Golz, J. (2008). Classification of apparent motion percepts based on temporal factors. Journal of Vision, 8, 1-22.

Farrell, J. E. (1983). Visual transformations underlying apparent movement. Perception \& Psychophysics, 33, 85-92.

Farrrell, J. E., \& Shepard, R. N. (1981). Shape, orientation, and apparent rotational motion: Journal of Experimental Psychology: Human Perception and Performance, 7, 477-486.

Feldman, J., \& Tremoulet, P. (2006). Individuation of visual objects over time. Cognition, 99, 131-165.

Flombaum, J. I., \& Scholl, B. J. (2006). A temporal same-object advantage in the tunnel effect: Facilitated change detection for persisting objects: Journal of Experimental Psychology: Human Perception and Performance, 32, 840-853.
Foster, D. H. (1975). Visual apparent motion of some preferred paths in the rotation group $\mathrm{SO}(3)$. Biological Cybernetics, 18, $81-89$.

Freyd, J. J. (1987). Dynamic mental representations. Psychological Review, 94, 427-438.

Freyd, J. J., \& Finke, R. A. (1984). Representational momentum. Journal of Experimental Psychology: Learning, Memory, and Cognition, 10, 126-132.

Freyd, J. J., Pantzer, T. M., \& Cheng, J. L. (1988). Representing statics as forces in equilibrium. Journal of Experimental Psychology: General, 117, 395-407.

Gerbino, W. (1984). Low-level and high-level processes in the perceptual organization of three-dimensional apparent motion. Perception, 13, 417-428.

Gerbino, W., \& Salmaso, D. (1987). The effect of amodal completion on visual matching. Acta Psychologica, 65, 25-46.

Gibson, J. J. (1979). The ecological approach to visual perception. Boston: Houghton Mifflin.

Gibson, J. J., Kaplan, G. A., Reynolds, H. N., \& Wheeler, K. (1969). The change from visible to invisible: A study of optical transitions. Perception \& Psychophysics, 5, 113-116.

He, S., Cavanagh, P., \& Intriligator, J. (1997). Attentional resolution. Trends in Cognitive Science, 1, 115-121.

He, Z. J., \& Nakayama, K. (1994). Perceiving textures: Beyond filtering. Vision Research, 34, 151-162.

Hubbard, T. L. (2001). The effect of height in the picture plane on the forward displacement of ascending and descending targets. Canadian Journal of Experimental Psychology, 55, 325-330.

Hubbard, T. L., \& Ruppel, S. E. (2000). Spatial memory averaging, the landmark attraction effect, and representational gravity. Psychological Research, 59, 41-55.

Kahneman, D. (1967). An onset-onset law for one case of apparent motion and metacontrast. Perception \& Psychophysics, 2, $577-$ 584 .

Kahneman, D., \& Wolman, R. E. (1970). Stroboscopic motion: Effects of duration and interval. Perception \& Psychophysics, 8, 161164.

Kanizsa, G. (1979). Organization in vision. New York: Praeger.

Kanizsa, G., \& Gerbino, W. (1982). Amodal completion: Seeing or thinking? In J. Beck (Ed.), Organization and representation in perception (pp. 167-190). Hillsdale: Erlbaum.

Kaplan, G. A. (1969). Kinetic disruption of optical texture: The perception of depth at an edge. Perception \& Psychophysics, 6, 193-198.

Kawachi, Y., \& Gyoba, J. (2006). A new response-time measure of object persistence in the tunnel effect. Acta Psychologica, 123, 73-90.

Kellman, P. J., \& Shipley, T. F. (1991). A theory of visual interpolation in object perception. Cognitive Psychology, 23, 141-221.

Kohler, A., Haddad, L., Singer, W., \& Muckli, L. (2008). Deciding what to see: The role of intention and attention in the perception of apparent motion. Vision Research, 48, 1096-1106.

Kolers, P. A. (1972). Aspects of motion perception. New York: Pergamon.

Kolers, P. A., \& Pomerantz, J. R. (1971). Figural change in apparent motion. Journal of Experimental Psychology, 87, 99-108.

Korte, A. (1915). Kinematoskopische Untersuchungen [Cinematoscopic investigations]. Zeitschrift für Psychologie, 72, 193-296.

Marr, D. (1982). Vision. New York: Freeman.

McBeath, M. K., \& Shepard, R. N. (1989). Apparent motion between shapes differing in location and orientation: A window technique for estimating path curvature. Perception \& Psychophysics, 46, 333-337.

Michotte, A., Thinès, G., \& Crabbé, G. (1964/1991). Amodal completion of perceptual structures. In G. Thinès, A. Costall, \& 
G. Butterworth (Eds.), Michotte's experimental phenomenology of perception (pp. 140-167). Hillsdale: Erlbaum. Original work published 1964.

Nagai, M., Kazai, K., \& Yagi, A. (2002). Larger forward memory shift in the direction of gravity. Visual Cognition, 9, 28-40.

Nakayama, K., He, Z. J., \& Shimojo, S. (1995). Visual surface representation: A critical link between lower-level and higher-level vision. In S. M. Kosslyn \& D. N. Osheron (Eds.), Invitation to cognitive science (pp. 1-70). Cambridge: MIT Press.

Pelli, D. G. (1997). The VideoToolbox software for visual psychophysics: Transforming numbers into movies. Spatial Vision, 10, 437-442.

Ramachandran, V. S., \& Anstis, S. M. (1983). Perceptual organization in moving patterns. Nature, 304, 529-531.

Ramachandran, V. S., Inada, V., \& Kiama, G. (1986). Perception of illusory occlusion in apparent motion. Vision Research, 26, 1741-1749.

Rauschenberger, R., Peterson, M. A., Mosca, F., \& Bruno, N. (2004). Amodal completion in visual search. Psychological Science, 15, 351-355.

Reed, C. L., \& Vinson, N. G. (1996). Conceptual effects on representational momentum. Journal of Experimental Psychology. Human Perception and Performance, 22, 839-850.

Reichardt, W. (1961). Autocorrelation, a principle for the evaluation of sensory information by the central nervous system. In W. A. Rosenblith (Ed.), Sensory communication (pp. 303-318). New York: Wiley.

Rock, I. (1983). The logic of perception. Cambridge: MIT Press.

Scherzer, T. R., \& Ekroll, V. (2009). Intermittent occlusion enhances the smoothness of sampled motion. Journal of Vision, 9, 1-18.

Scholl, B. J., \& Pylyshyn, Z. W. (1999). Tracking multiple items through occlusion: Clues to visual objecthood. Cognitive Psychology, 38, 259-290.
Sekuler, A. B., Palmer, S. E., \& Flynn, C. (1994). Local and global processes in visual completion. Psychological Science, 5, 260 267.

Shepard, R. N. (1984). Ecological constraints on internal representation: Resonant kinematics of perceiving, imagining, thinking, and dreaming. Psychological Review, 91, 417-447.

Shepard, R. N., \& Judd, S. A. (1976). Perceptual illusion of rotation of three-dimensional objects. Science, 191, 952-954.

Shepard, R. N., \& Zare, S. L. (1983). Path-guided apparent motion. Science, 220, 632-634.

Shiffrar, M., \& Freyd, J. (1990). Apparent motion of the human body. Psychological Science, 1, 257-264.

Shimojo, S., \& Nakayama, K. (1990). Amodal presence of partially occluded surfaces: Role of invisible stimuli in apparent motion correspondence. Perception, 19, 285-299.

Sigman, E., \& Rock, I. (1974). Stroboscopic movement based on perceptual intelligence. Perception, 3, 9-28.

Singh, M. (2004). Modal and amodal completion generate different shapes. Psychological Science, 15, 454-459.

Watanabe, T. (1995). Orientation and color processing for partially occluded objects. Vision Research, 5, 647-655.

Werkhoven, P., Snipe, H. P., \& Koenderink, J. J. (1990). Effects of element orientation on apparent motion perception. Perception \& Psychophysics, 47, 509-525.

Wertheimer, M. (1912/1961). Experimental studies on the seeing of motion. In T. Shipley (Ed.), Classics in psychology (pp. 1032-1088). New York: Philosophical Library. Original work published 1912.

Yantis, S. (1995). Perceived continuity of occluded visual objects. Psychological Science, 6, 182-186.

Yantis, S., \& Nakama, T. (1998). Visual interactions in the path of apparent motion. Nature Neuroscience, 1, 508-512.

Yonas, A., Craton, L. G., \& Thompson, W. B. (1987). Relative motion: Kinetic information for the order of depth at an edge. Perception \& Psychophysics, 41, 53-59. 